\title{
Blood-brain barrier permeability of Gualou Guizhi granules and neuroprotective effects in ischemia/reperfusion injury
}

\author{
HUANG LI ${ }^{1}$, MIAO YE ${ }^{2}$, YUQIN ZHANG ${ }^{1}$, MINGQING HUANG $^{1}$, WEI XU ${ }^{1}$, \\ KEDAN CHU $^{1}$, LIDIAN CHEN ${ }^{3}$ and JINHUA QUE ${ }^{1}$ \\ ${ }^{1}$ Pharmacy College; ${ }^{2}$ Centre of Biomedical Research and Development; ${ }^{3}$ Rehabilitation Medicine College, \\ Fujian University of Traditional Chinese Medicine, Fuzhou, Fujian 350122, P.R. China
}

Received August 5, 2014; Accepted January 21, 2015

DOI: $10.3892 / \mathrm{mmr} .2015 .3520$

\begin{abstract}
The present study aimed to estimate the blood-brain barrier (BBB) permeability of Gualou Guizhi granules (GLGZG) in normal rats and in rat models of ischemia/reperfusion (I/R) injury, and to examine the neuroprotective effects of GLGZG. A sensitive high-performance liquid chromatography-quadrupole-time of flight-mass spectrometry analytical method was developed to determinate the components of GLGZG in the plasma and brain tissue. Middle cerebral artery occlusion (MCAO) in rats served as a model of in vivo I/R. Citrulline, gallic acid, albiflorin, peoniflorin, liquiritin apioside, liquiritin, isoliquiritin apioside, isoliquiritin, liquiritigenin, isoliquiritigenin and glycyrrhizinic acid rapidly passed into the bloodstream. Citrulline, albiflorin, peoniflorin, liquiritin apioside, liquiritin, liquiritigenin, isoliquiritigenin and glycyrrhizinic acid also passed the $\mathrm{BBB}$ and reached the brain tissue of MCAO rats, while isoliquiritigenin and glycyrrhizinic acid were not detected in the brain tissue of the normal rats. The potential neuroprotective effect of GLGZG was determined in MCAO rats. The intragastric administration of GLGZG following reperfusion of rats for $2 \mathrm{~h}$ decreased the neurological defects and infarction volume, attenuated pathological changes of brain tissue and exerted a significant protective effect in cerebral ischemia injury. In conclusion, certain components of GLGZG passed through the BBB, particularly following cerebral ischemia injury, and this may be therapeutically effective for the treatment of cerebral ischemia injury in the human brain.
\end{abstract}

Correspondence to: Dr Lidian Chen, Rehabilitation Medicine College, Fujian University of Traditional Chinese Medicine, 1 Qiuyang Road, Fuzhou, Fujian 350122, P.R. China

E-mail: clidianlab@gmail.com

Key words: Gualou Guizhi granule, blood-brain barrier, middle cerebral artery occlusion, neuroprotection, high-performance liquid chromatography-quadrupole-time of flight-mass spectometry

\section{Introduction}

Stroke, with ischemia of the brain and hemorrhagic injury as the predominant clinical manifestations, is the main cause of mortality worldwide (1-3). The annual number of stroke cases is increasing as the world population is ageing. According to the World Health Organization, 15,000,000 individuals suffer a stroke worldwide each year, which undoubtedly creates an increased financial and social burden to the surviving population (4). There are currently few preventative therapies available for stroke and the only Food and Drug Administration approved 'pharmacological' intervention to reduce brain damage is tissue plasminogen activator (5). However, only $\sim 3-5 \%$ of patients benefit from this, due to its narrow time window.

The blood-brain barrier (BBB) is a highly selective permeability barrier, which separates the circulating blood from the brain extracellular fluid in the central nervous system (6). The BBB allows the passage of water, certain gases and lipid soluble molecules by passive diffusion, and the selective transport of molecules, including glucose and amino acids, which are crucial to neurological function (6). By contrast, the BBB may prevent the entry of lipophilic, potential neurotoxins using an active transport mechanism, which is important for protecting the brain from fluctuations in the plasma composition and from circulating agents (7).

Changes in the permeability of the BBB, which result in the increase of vascular-derived substances into the brain, leading to pathophysiologic processes and affecting the passage of drugs and various metabolites into the brain parenchyma, have been investigated $(8,9)$.

Gualou Guizhi granules (GLGZG; approval no. S20130001) are a standard prescribed drug at Fujian University of Traditional Chinese Medicine (TCM) Affiliated Second People's Hospital (Fuzhou, China). It is a well-known traditional Chinese formula, first recorded in 'Essentials from the Golden Cabinet' (10). GLGZG is comprised of six herbs, including Trichosanthes kirilowii Maxim., Paeonia lactiflora Pall., Cinnamomum cassia Presl., Glycyrrhiza uralensis Fisch., Zingiber officinale Rosc. and Ziziphus jujuba Mill., according to the Yin-Yang and Wu Hsing 'Five Elements' theory of TCM, in a weight ratio of 10:3:3:3:2:3 (10). It has been used clinically to treat muscular spasticity following 
stroke, epilepsy and spinal cord injury in China (11-13). Our previous clinical study demonstrated beneficial effects of GLGZG in stroke patients (unpublished data) and our previous studies evaluated the effect of GLGZG on lipopolysaccharide (LPS)-induced BV-2 murine microglial cells and demonstrated that GLGZG had an effect on the Toll-like receptor (TLR)4/nuclear factor (NF)- $\kappa$ B pathway and mitogen-activated protein kinase (MAPK) signaling pathway $(14,15)$. The neuroprotective effects of GLGZG on glutamate-induced apoptosis in cultured BV-2 cells (16) and the neuroprotective effects of GLGZG in vivo were investigated, which demonstrated that GLGZG exerts its neuroprotective effects via the modulation of excitatory amino acid levels and the expression of N-methyl-D-aspartate (NMDA) and $\alpha$-amino-3-hydroxy-5 -methyl-4-isoxazolepropionic acid (AMPA) receptors $(17,18)$.

Although previous studies have demonstrated the neuroprotective effect of GLGZG in vivo and in vitro, the BBB permeability of GLGZG under normal or ischemia/reperfusion (I/R) injury remains to be elucidated. The permeability of the BBB is an important factor for compounds used in the treatment of neurodegenerative disorders. The present study investigated the BBB permeability of GLGZG in normal and I/R injury models in vivo and also further examined the neuroprotective effects of GLGZG in vivo.

\section{Materials and methods}

Materials. GLGZG was provided by Fujian University of TCM Affiliated Second People's Hospital (Fuzhou, China). High-performance liquid chromatography (HPLC) grade methanol and acetonitrile were purchased from Merck Co. (Darmstadt, Germany). Standard substances, including citrulline, gallic acid, albiflorin, peoniflorin, liquiritin apioside, liquiritin, isoliquiritin apioside, isoliquiritin, liquiritigenin, isoliquiritigenin and glycyrrhizinic acid were purchased from the National Institute for the Control of Pharmaceutical and Biological Products (Beijing, China). All other chemical reagents used were of analysis grade.

A total of 98 male Sprague-Dawley rats (4 weeks old; weighing $280 \pm 20 \mathrm{~g}$ ) were obtained from the Laboratory Animal Center of Fujian University of TCM. The animals were housed under controlled temperature conditions $\left(21-23^{\circ} \mathrm{C}\right)$ with a relative humidity of $55 \pm 5 \%$, a 12 -h light/dark cycle and free access to standard rat diet and tap water. The study was approved by the ethics committee of the Animal Experimental Center, Fujian University of TCM (Fuzhou, China).

Model of focal cerebral I/R. An intraluminal suture was used for the induction of focal cerebral ischemia, as described in Longa et al (17-19), with modification. Briefly, the rats were anesthetized using $10 \%$ chloral hydrate solution (0.3 ml/100 g, intraperitoneally administered; Sinopharm Chemical Reagent Co., Ltd., Shanghai, China) and the left common carotid artery (CCA), external carotid artery (ECA) and internal carotid artery (ICA) were exposed by a short incision, and separated from the adjacent nerves and tissue. A 3-0 silicon rubber-coated nylon monofilament (Guangzhou Jialing Biotechnology Co., Ltd., Guangzhou, China) was carefully inserted into the ICA and was advanced to occlude the origin of the left middle cerebral artery (MCAO) until light resistance was detected at $18-20 \mathrm{~mm}$ from the CCA bifurcation. Following ischemia for $2 \mathrm{~h}$, the filament was withdrawn allowing reperfusion. Neurological defects were scored using a five-point scale: 0 , no neurological symptoms; 1 , unable to completely extend the front jaw on the other side; 2 , rotating while crawling and falling to the contralateral side; 3 , unable to walk without assistance; and 4, unconsciousness (16). Rats with a score of 1-3 points were considered successful models of $\mathrm{I} / \mathrm{R}$ and were used in the subsequent experiments.

Analysis of the compounds in the plasma and brain by HPLC-quadrupole-time of flight-mass spectrometry $(Q-T O F-M S)$. A total of 15 rats were separated into a normal group, a normal group with GLGZG treatment and an I/R model group with GLGZG treatment. The rats in the normal group were subjected to the surgical procedure with exposure of the ICA and ECA, however, no MCAO was induced, and the rats received normal saline treatment. The rats in the normal group with GLGZG treatment received GLGZG (7.2 g/kg/day intragastrically), however, were not exposed to MCAO surgery. The model group with GLGZG treatment received GLGZG (7.2 g/kg/day intragastrically) and underwent MCAO surgery. Following treatment with GLGZG for 15,30 or 60 min (5 rats at each time point), the rats were deeply anesthetized with $10 \%$ chloral hydrate solution $(0.3 \mathrm{ml} / 100 \mathrm{~g}$, intraperitoneally administered) and decapitated, then brains were resected immediately, prior to plasma and brain tissue samples being obtained. The brain tissue was homogenized in deionized water at a ratio of 1:1 (w/v) using a glass pestle, and the homogenate was centrifuged at $628 \mathrm{x}$ g for $15 \mathrm{~min}$ at $4^{\circ} \mathrm{C}$ to obtain the supernatant. Methanol (4-fold) was then added to the brain tissue homogenate or plasma samples. The samples were then vortex mixed for $2 \mathrm{~min}$, followed by ultrasound-associated extraction using a KQ-500DE single-frequency ultrasonic cleaner (Kunshan Ultrasonic Instruments Co., Ltd., Kunshan, China) for $10 \mathrm{~min}$. Following centrifugation at $628 \mathrm{x} \mathrm{g}$ for $10 \mathrm{~min}$ at $4^{\circ} \mathrm{C}, 2 \mu \mathrm{l}$ supernatant was injected into the HPLC system.

Chromatographic analysis was performed on a Shimadzu HPLC system (Shimadzu Corporation, Kyoto, Japan) coupled with a Bruker micrOTOF-Q II mass spectrometer (Bruker Daltonik, Bremen, Germany). The HPLC system consisted of an LC-20A pump, DGU-20A ${ }_{5}$ degasser, SIL-20A auto-injector and CTO-20A column thermostat. The mobile phases consisted of $0.1 \%$ formic acid (Aladdin Industrial Corporation, Shanghai, China) in (A) water and (B) acetonitrile high performance liquid chromatography grade (Merck Millipore, Darmstadt, Germany). The gradient elution program was as follows: $15 \%$ B for 0-18 min; 15-48\% B for 18-20 min; 48-15\% B for 20-25 min. Another chromatographic separation was performed at a flow rate of $0.3 \mathrm{ml} / \mathrm{min}$ using a sample volume of $2 \mu \mathrm{l}$ and a Shimadzu Inertsil SP C18 $5 \mu \mathrm{m}(250 \times 4.6 \mathrm{~mm})$ column (Shimadzu, Kyoto, Japan).

The microTOF-Q II mass spectrometer was equipped with an electrospray ionization source and run in negative mode. The acquisition parameters of Q-TOF-MS were as follows: Capillary, $-4.5 \mathrm{kV}$ (negative mode); nebulizer pressure, 2.0 bar; dry gas $\left(\mathrm{N}_{2}\right)$ flow rate, $4.0 \mathrm{l} / \mathrm{min}$; dry gas temperature, $180^{\circ} \mathrm{C}$. Funnel 1 and 2 were $200.0 \mathrm{Vpp}$; hexapole Rf, $100.0 \mathrm{Vpp}$; 


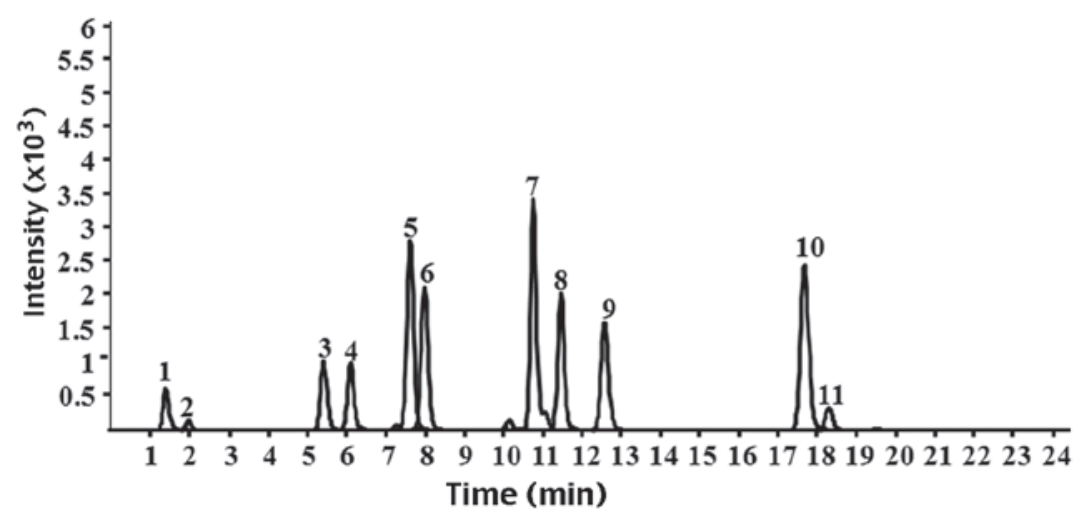

Figure 1. Chromatograms of the nine standard reference compounds in high-performance liquid chromatography-quadrupole-time of flight-mass spectrometry. 1, citrulline; 2, gallic acid; 3, albiflorin; 4, peoniflorin; 5, liquiritin apioside; 6, liquiritin; 7, isoliquiritin apioside; 8, isoliquiritin; 9, liquiritigenin; 10, isoliquiritigenin; 11, glycyrrhizinic acid.

quadrupole ion energy, $2.5 \mathrm{eV}$; collision Rf, $150.0 \mathrm{Vpp}$. The ion transfer time was $80 \mu \mathrm{sec}$ and the prepulse storage time was $5 \mu \mathrm{sec}$. Argon (Fuzhou Huaxin Industrial Gases Co., Ltd., Fuzhou, China) was applied as the collision gas and the collision energy were set between 20 and $50 \mathrm{eV}$ to obtain the fragment ions data. To obtain highly accurate mass values, external calibration of the Q-TOF/MS was performed daily prior to sample injection, and the enhanced quadratic calibration mode was used for the calibration curve.

\section{Neuroprotection of GLGZG in I/R injury}

Experimental grouping and treatment. A total of 48 rats were divided into the three experimental groups, described above and treatment was performed, as described below. The experimental animals were grouped as: Sham-operated group $(n=16)$, in which the rats were subjected to the surgical procedure to expose the ICA and ECA, however, no MCAO was induced and the rats received normal saline; an MCAO model group $(\mathrm{n}=16)$, in which the rats received normal saline and underwent MCAO surgery; and a GLGZG group $(\mathrm{n}=16)$, in which the rats received GLGZG treatment $(7.2 \mathrm{~g} / \mathrm{kg})$ and underwent MCAO surgery. The rats were treated daily for 1 week.

Scoring neurological defects. The neurobehavioral defects of the rats were quantified using the five-point scale, as described above (16), and this evaluation was performed in a blinded-manner by Miss. Yuqin Zhang.

2,3,5-triphenyltetrazolium chloride (TTC) staining. A total of eight rats from each group were sacrificed using $10 \%$ chloral hydrate and perfused transcardiacally with $0.9 \% \mathrm{NaCl}$ prior to decapitation and rapid removal of the brain. The brains were stored at $20^{\circ} \mathrm{C}$ for $5 \mathrm{~min}$ and then dissected into six coronal slices $(2 \mathrm{~mm})$ continuously from front to back. The brain tissues were immersed in 2\% TTC staining solution (cat no. T8877; Sigma-Aldrich, St. Louis, MO, USA) in phosphate-buffered saline (pH 7.4; Beyotime Institute of Biotechnology, Haimen, China), and incubated at $37^{\circ} \mathrm{C}$ for $1 \mathrm{~h}$ and turned over six times. A high-resolution digital camera (IXUS130; Canon, Inc., Tokyo, Japan) was used to capture images and a Motic Med 6.0 Digital Medical Image Analysis system (Motic Instruments Inc., Richmond, Canada) was used to calculate the infarct volume as a percentage of the viable cerebral tissue of the entire brain (20).
Cerebral histopathology. A total of eight rats from each group were sacrificed using $10 \%$ chloral hydrate and perfused transcardiacally with $4 \%$ paraformaldehyde solution and $0.9 \% \mathrm{NaCl}$ (Beyotime Institute of Biotechnology), following which the brains were rapidly removed. The brains were paraffin-embedded (Beyotime Institute of Biotechnology), prior to being dissected into a series of adjacent $5 \mu \mathrm{m}$ thick sections and stained with hematoxylin and eosin (Beyotime Institute of Biotechnology). Histopathological changes were observed under a DM4000B light microscope (Leica Microsystems GmbH, Wetzlar, China).

Statistical analysis. The data are expressed as the mean \pm standard deviation. Analysis of variance was performed to determine significant differences between groups using SPSS 16.0 software (SPSS, Inc., Chicago, IL, USA). P<0.05 was considered to indicate a statistically significant difference.

\section{Results}

Analysis of the compounds in the plasma and brain using HPLC-Q-TOF-MS. The standard components of GLGZG, including citrulline, gallic acid, albiflorin, peoniflorin, liquiritin apioside, liquiritin, isoliquiritin apioside, isoliquiritin, liquiritigenin, isoliquiritigenin and glycyrrhizinic acid were detected by HPLC-Q-TOF-MS (Fig. 1). The result demonstrated no peaks in the chromatograms of the blank plasma and blank brain tissue homogenate. Citrulline, albiflorin, peoniflorin, liquiritin apioside, liquiritin, isoliquiritin apioside, isoliquiritin, liquiritigenin, isoliquiritigenin and glycyrrhizinic acid were present in the plasma from the rat model of MCAO and the normal rats. Only citrulline, gallic acid, albiflorin, peoniflorin, liquiritin apioside, liquiritin, liquiritigenin, isoliquiritigenin and glycyrrhizinic acid were detected in the brain tissue homogenates from the rat models of MCAO by HPLC-Q-TOF-MS. However, gallic acid, isoliquiritigenin and glycyrrhizinic acid were not detected in the brain tissue homogenate from the normal rat group compared with the rat model of MCAO. Representative chromatograms of plasma and brain tissue homogenate are shown in Figs. 2 and 3, respectively. 
A

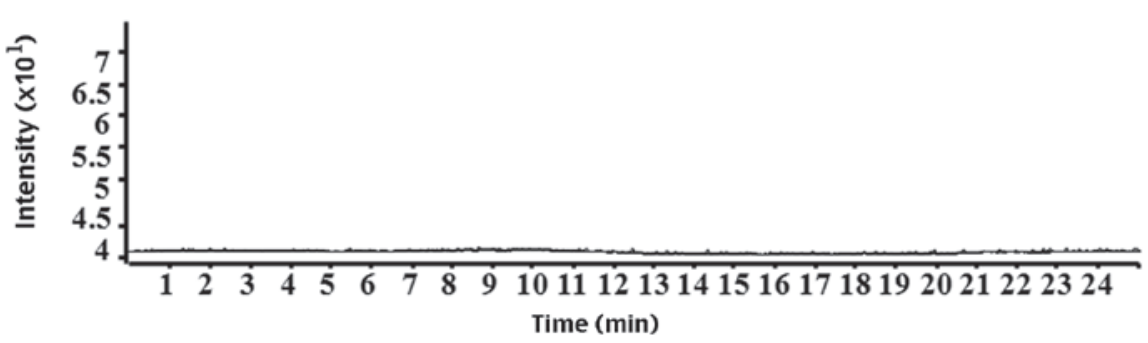

B

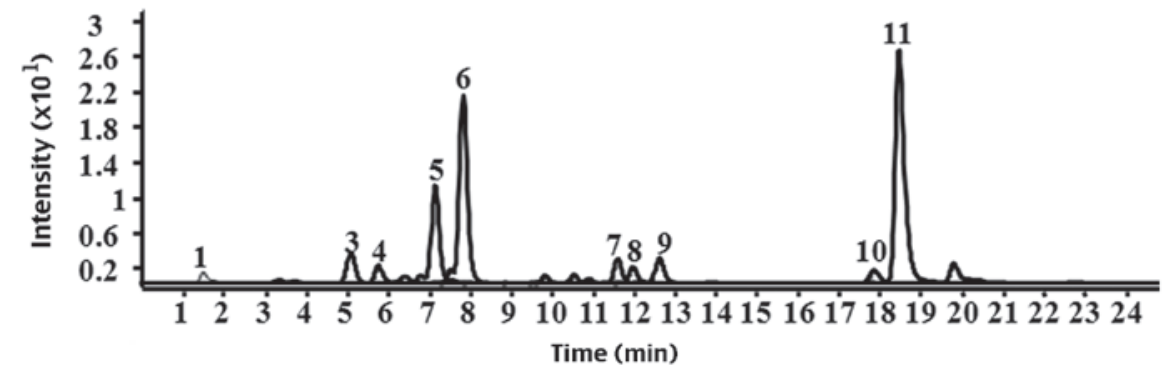

C

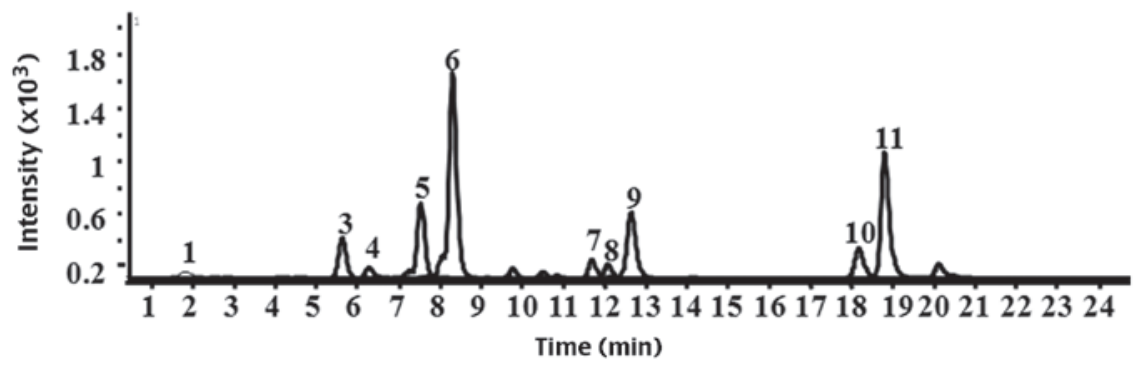

Figure 2. Chromatograms of plasma following treatment with Gualou Guizhi granule. (A) Blank plasma, (B) plasma from normal rats and (C) plasma from rat models of middle cerebral artery occlusion. 1, citrulline: 3 , albiflorin; 4, peoniflorin; 5, liquiritin apioside; 6 , liquiritin; 7, isoliquiritin apioside; 8 , isoliquiritin; 9, liquiritigenin; 10, isoliquiritigenin; 11, glycyrrhizinic acid.

Treatment with GLGZG improves the neurological deficits and attenuates cerebral infarct volume in rats. The effect of GLGZG on neurological deficits was evaluated by measuring the neurological performance using a five-point scale, as described above. As shown in Fig. 4, the rats in the sham-operated group (score: 0 ) exhibited no neurological deficits, while the rats in the MCAO model group exhibited neurological deficit (score: 1-3), including circling towards the contralateral side with reduced mobility, inability to completely extend the front jaw on the other side, rotating while crawling and falling to the contralateral side. The rats in the GLGZG treated group exhibited significantly improved neurological symptoms between days 1 and 7, particularly those in the high-dose group.

The infarct volumes of the rats were also determined to evaluate the impact of GLGZG treatment for 7 days following MCAO. As shown in Fig. 5A and B, the mean infraction volume in the MCAO model group was significantly higher compared with the sham-operated group. Treatment with GLGZG reduced the infarct volume to $12 \pm 1.2 \%$ compared with that of the MCAO model group.

Treatment with GLGZG improves cerebral histopathology. As shown in Fig. 6, in the sham-operated group, the brain tissue remained intact, neurons maintained eumorphism and uniform distribution, cytoplasms were pale pink and abundant and no inflammatory cell infiltrate was observed. By contrast, the brain tissues had a larger infarct area, neurons were disordered and reduced, with increased volume and staining in the cytoplasms was shallower with vacuolization in the MCAO model group. However, following treatment with GLGZG, the infarct area was markedly reduced, the extent of damage was decreased significantly and cytoplasmic hypervacuolization was reduced.

\section{Discussion}

GLGZG is a well-known traditional Chinese formula, which was first recorded in 'Essentials from the Golden Cabinet' in 210 AD. It is now developed into granules as an easy-to-use form (10). In order to investigate the efficacy and mechanism underlying GLGZG, our previous studies evaluated the effect of GLGZG on LPS-induced BV-2 murine microglial cells and demonstrated that GLGZG has an inhibitory effect on the TLR4/NF- $\kappa B$ (14) and the MAPK signaling pathways (15). The neuroprotective effects of GLGZG on glutamate-induced apoptosis in cultured BV-2 cells was also investigated (16). In addition, to confirm the results observed in vitro, a reversible model of focal ischemia (MCAO model) was used to induce a model of focal brain ischemia in vivo. It was revealed that GLGZG exerts neuroprotective effects via the modulation of excitatory amino acid levels and the expression levels of the NMDA and AMPA receptors $(17,18)$. Although possible mechanisms have been identified, it is known that the BBB, a diffusion barrier, is responsible for strictly controlling the exchanges between the blood and brain compartments (6), 
A

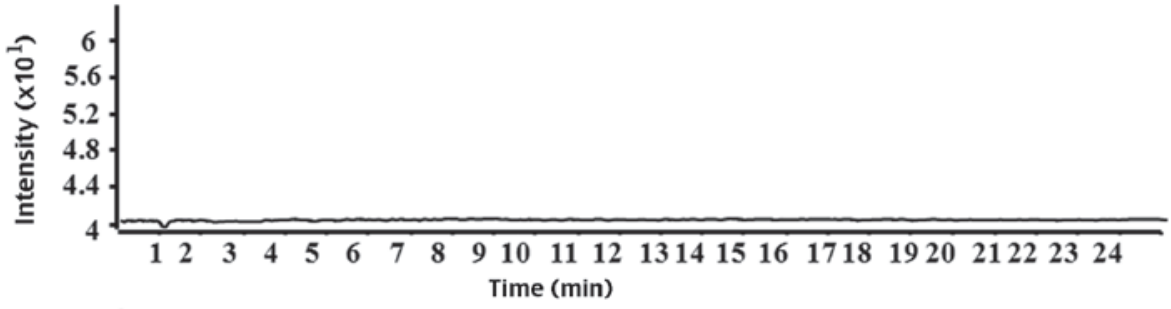

B

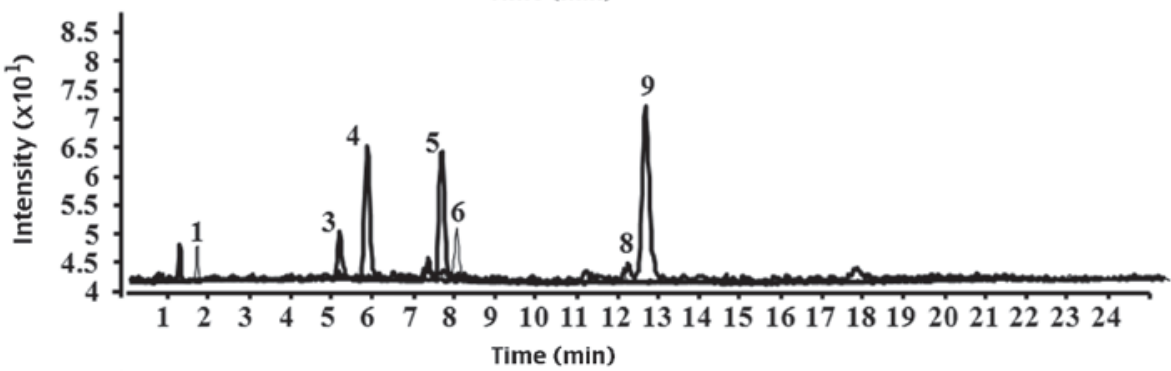

C

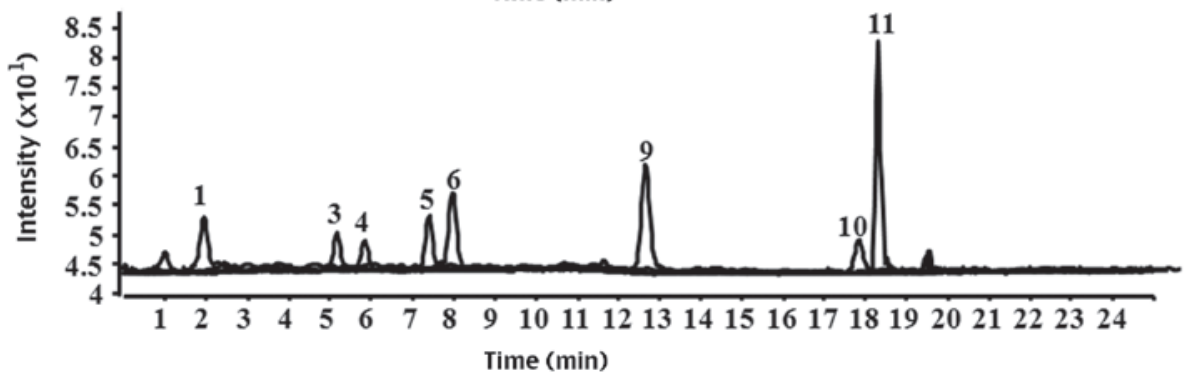

Figure 3. Chromatograms of brain tissue homogenate following treatment with Gualou Guizhi granule. (A) Brain tissue homogenate, (B) brain tissue homogenate from normal rats and (C) brain tissue homogenate from rat models of middle cerebral artery occlusion. 1, citrulline; 2, gallic acid; 3, albiflorin; 4, peoniflorin; 5, liquiritin apioside; 6, liquiritin; 9, liquiritigenin; 10, isoliquiritigenin; 11, glycyrrhizinic acid.

whether the $\mathrm{BBB}$ changes following cerebral $\mathrm{I} / \mathrm{R}$ remains to be elucidated. Whether the $\mathrm{BBB}$ alters following cerebral $\mathrm{I} / \mathrm{R}$ injury, and which compounds of GLGZG penetrate the BBB, remain to be fully elucidated.

During I/R, the brain environment may be altered. Loss of blood supply to the brain results in a cascade of events throughout the infarcted region, including depletion of adenosine triphosphate, excitotoxic glutamate efflux, a neuronal component, ionic imbalance, including increased intracellular calcium, loss of metabolic function with increased acidosis, oxidative stress and activation of the inflammatory processes (8). Ultimately, this causes the destruction and/or dysfunction of brain cells, which leads to clinically definable neurological deficits (6).

The present study confirmed the neuroprotective effects of GLGZG in I/R injury, including decreased neurological defects and infarct volume, and ameliorated cerebral histopathology. Together with previous findings, these results suggested that GLGZG is a promising alternative therapeutic approach.

Under normal physiological conditions, the BBB prevents the paracellular diffusion of hydrophilic solutes and the efflux of hydrophobic molecules and drugs from the brain to the blood by active transport (6). However, the BBB is disrupted following ischemic stroke and the subsequent onset of reperfusion. This affects the passage of drugs and various metabolites into the brain (21). Therefore, penetration of the BBB by therapeutic agents is a prerequisite for treatment of central nervous diseases. The present study aimed to investigate the BBB permeability of GLGZG using HPLC-Q-TOF-MS analysis to detect compounds in the brain tissues of normal rats and rat models of $\mathrm{I} / \mathrm{R}$ injury in vivo.

The present study synchronously measured the compounds of GLGZG in the plasma and brain tissue from normal and model rats. Citrulline, albiflorin, peoniflorin, liquiritin apioside, liquiritin, isoliquiritin apioside, isoliquiritin, liquiritigenin, isoliquiritigenin and glycyrrhizinic acid were detected in the blood of normal and model rats. Citrulline, gallic acid, albiflorin, peoniflorin, liquiritin apioside, liquiritin, isoliquiritin apioside, isoliquiritin, liquiritigenin, isoliquiritigenin and glycyrrhizinic acid were detected in the brain tissue from model rats, however, only citrulline, albiflorin, peoniflorin, liquiritin apioside, liquiritin and liquiritigenin were detected in the brain tissue from normal rats. Compounds with high lipid solubility and low relative molecular mass penetrate the $\mathrm{BBB}$ more easily than others (22), and it was demonstrated that the detected compounds in the brain tissue from normal rats conformed to these characteristics. However, this was not observed in the brain tissues from the model rats. Glycyrrhizinic acid, with a relative molecular mass of 822.92 , was detected in brain tissue from the model rats, but not the normal rats and gallic acid, isoliquiritigenin and glycyrrhizinic acid were increased in the brain tissues from the model rats compared with the normal rats, suggesting that these compounds were able to penetrate the $\mathrm{BBB}$ rapidly and distribute into the brain tissue as a result of BBB damage.

No previous investigations, to the best of our knowledge, have demonstrated the penetration of glycyrrhizinic acid into 


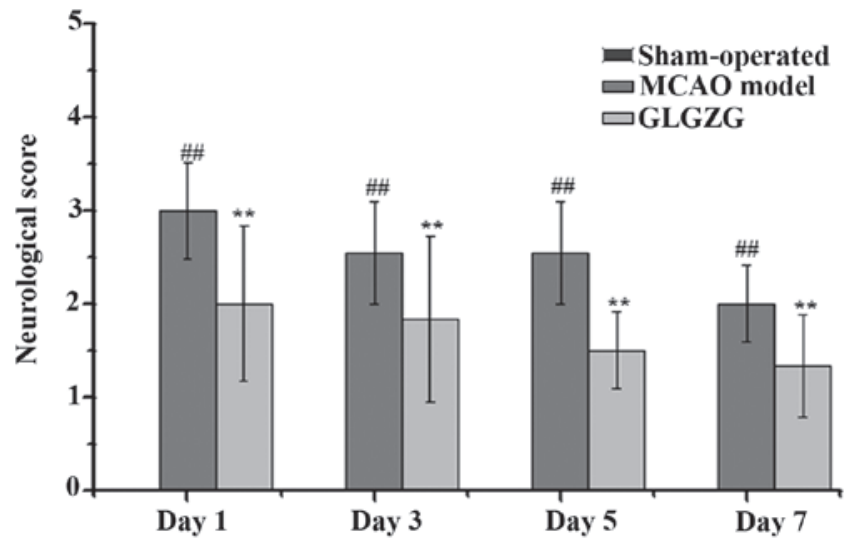

Figure 4. Effects of treatment with GLGZG on neurological deficits. The neurological score of the sham-operated, MCAO group and GLGZG groups are shown $(\mathrm{n}=16)$. The Sham-operated rats underwent surgery without vessel occlusion; MCAO rats were treated with saline for 7 days following transient MCAO; GLGZG rats were treated with $7.2 \mathrm{~g} / \mathrm{kg}$ GLGZG for 7 days following transient MCAO. Data are expressed as the mean \pm standard deviation ( ${ }^{* *} \mathrm{P}<0.01$ vs. $\mathrm{MCAO}$ and ${ }^{\# \#} \mathrm{P}<0.01$ vs. sham-operation). GLGZG, Gualou Guizhi granule; MCAO, middle cerebral artery occlusion.

A

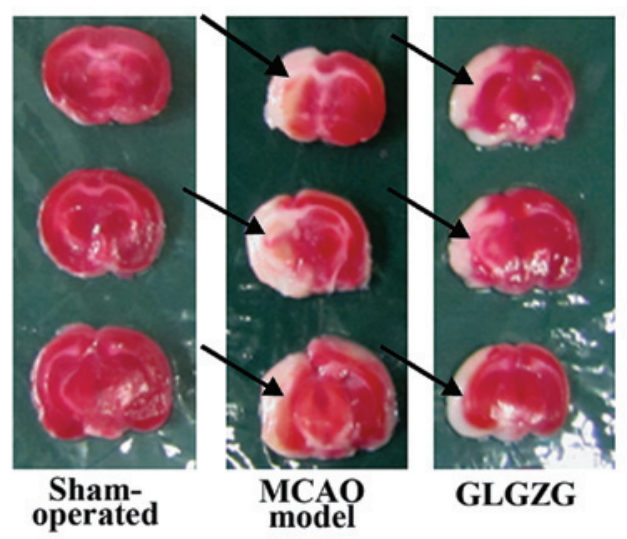

B

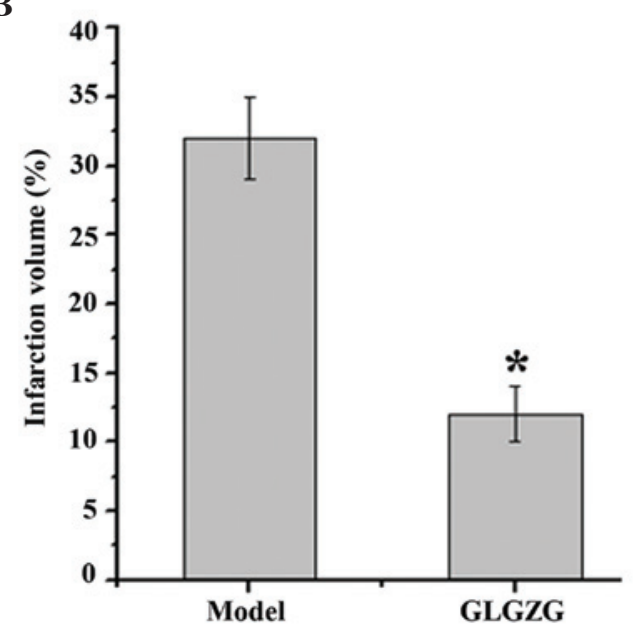

Figure 5. Effects of GLGZG on the size of cerebral infarcts. (A) Representative images demonstrating the cerebral infarct of the rat brain slices measured by 2,3,5-triphenyltetrazolium chloride staining $(\mathrm{n}=8)$. The black arrows indicate the 'infarcted areas'. (B) Infarct volume of the MCAO (model) group and GLGZG group. The sham-operated rats underwent surgery without vessel occlusion; MCAO rats were treated with saline for 7 days following transient MCAO; GLGZG rats were treated with $7.2 \mathrm{~g} / \mathrm{kg}$ GLGZG for 7 days following transient MCAO. Data are expressed as the mean \pm standard deviation ("P<0.01, vs. Model). GLGZG, Gualou Guizhi granule; MCAO, middle cerebral artery occlusion, Sham, surgery without vessel occlusion.

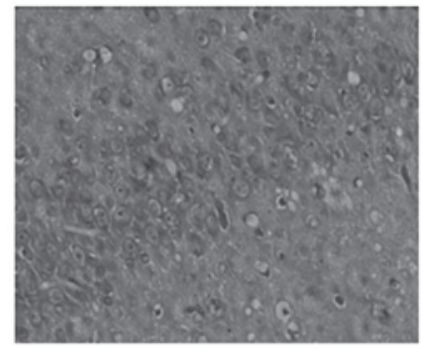

Sham-operated

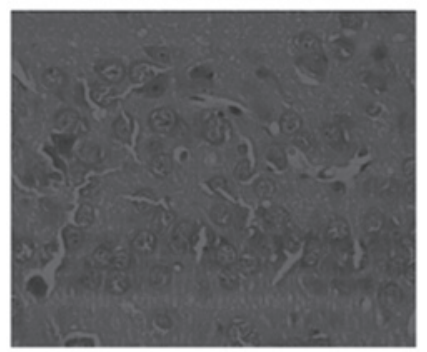

MCAO model

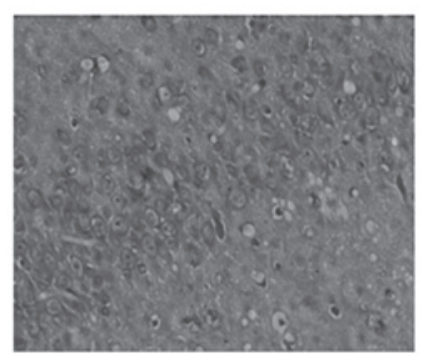

GLGZG

Figure 6. Effect of treatment with GLGZG on the histopathological changes of brain tissue following focal cerebral ischemic/reperfusion injury in rats. Representative images are shown. (hematoxylin and eosin stained; magnification, x200). The Sham-operated rats underwent surgery without vessel occlusion; MCAO rats were treated with saline for 7 days following transient MCAO; GLGZG rats were treated with $7.2 \mathrm{~g} / \mathrm{kg}$ GLGZG for 7 days following transient MCAO. GLGZG, Gualou Guizhi granule; MCAO, middle cerebral artery occlusion.

the brain. A previous study demonstrated that diammonium glycyrrhizinate ameliorates inflammation in focal cerebral $\mathrm{I} / \mathrm{R}$ injury (21). Isoliquiritigenin, a licorice chalconoid, is known to have vasorelaxant, antioxidant, antiplatelet, antitumor, anti-allergenic, antiviral and estrogenic properties, and Zhan and Yang demonstrated that isoliquiritigenin exhibited protective effects in transient MCAO-induced focal cerebral ischemia in rats $(23,24)$. Paeoniflorin, a monoterpene glucoside, suggested to exhibit several pharmacological effects attenuates ischemia-induced pathological and behavioral changes (25-27). Glycyrrhizin has been associated with numerous pharmacological effects and has been observed to have a protective effect on focal cerebral I/R-induced inflammation, oxidative stress and apoptosis in rats $(28,29)$. Additionally, the Institute of Cancer Research reported that liquiritin exerts a neuroprotective effect against focal cerebral $\mathrm{I} / \mathrm{R}$ in male mice (30).

GLGZG contains several herbs and is chemically complex, with hundreds of components. As mentioned above, certain bioactive chemicals have been suggested to penetrate the $\mathrm{BBB}$, enter the brain and collectively exert therapeutic and 
modulatory effects, providing a beneficial property with possible clinical use in the treatment of ischemic stroke.

In conclusion, the present study demonstrated that the BBB permeability of GLGZG increased significantly following I/R injury in vivo, with citrulline, gallic acid, albiflorin, peoniflorin, liquiritin apioside, liquiritin, liquiritigenin, isoliquiritigenin and glycyrrhizinic acid being detected in the brain following MCAO. In addition, GLGZG exhibited a protective effect in ischemic injury in rats.

\section{Acknowledgements}

This study was performed at the State Ley Laboratory of Chinese Pharmacies of Fujian Provincial Department of Science and Technology, Collaborative Innovation Center for Rehabilitation Technology and TCM Rehabilitation Research Center of the State Administration of Traditional Chinese Medicine. This study was funded by grants from the Important Subject of Fujian Province Science and Technology Hall of China (no. 2012Y0041) and the Fujian province Education Hall of China (no. JA12176).

\section{References}

1. Lloyd-Jones D, Adams RJ, Brown TM, et al: Executive summary: heart disease and stroke statistics - 2010 update: a report from the American Heart Association. Circulation 121: 948-954, 2010.

2. Liu M, Wu BO, Wang WZ, Lee LM, Zhang SH and Kong LZ: Stroke in China: epidemiology, prevention, and management strategies. Lancet Neurolo 6: 456-464, 2007.

3. Centers for Disease Control and Prevention (CDC): Prevalence of disabilities and associated health conditions among adults - United States, 1999. MMWR Morb Mortals Wkly Rep 50: 120-125, 2001.

4. The World Health Report 2002. World Health Organization. Geneva, Switzerland, 2002.

5. Del Zoppo GJ, Saver JL, Jauch EC, Adams HP Jr and American Heart Association Stroke Council: Expansion of the time window for treatment of acute ischemic stroke with intravenous tissue plasminogen activator: a science advisory from the American Heart Association/American Stroke Association. Stroke 40: 2945-2948, 2009.

6. Ballabh P, Braun A and Nedergaard M: The blood-brain barrier: an overview: structure, regulation, and clinical implications. Neurobiol Dis $16: 1-13,2004$.

7. Begley DJ and Brightman MW: Structural and functional aspects of the blood-brain barrier. Prog Drug Res 61: 39-78, 2003.

8. Sandoval KE and Witt KA: Blood-brain barrier tight junction permeability and ischemic stroke. Neurobiol Dis 32: 200-219, 2008.

9. Strbian D, Durukan A, Pitkonen M, Marinkovic I, Tatlisumak E, Pedrono E, et al: The blood-brain barrier is continuously open for several weeks following transient focal cerebral ischemia. Neuroscience 153: 175-181, 2008.

10. Zhang ZJ, Jinkui YL, Lin Y, Yang PJ, Hou XM and Yang YW: Synopsis of Golden Chamber. Macmillan Press, Beijing, China, pp203-204, 2008 (In Chinese).

11. Sun X: Research on formula treating paralysis and spasticity from 'treatise on febrile and miscellaneous diseases'. Zhongguo Zhong Yi Ji Chu Yi Xue Za Zhi 8: 644-645, 2010 (In Chinese).
12. Yang C, Chen L and Tao J: New usage of a classical formula-Gua Lou Gui Zhi decoction. Liaoning J Tradit Chin Med 8: 166-167 2010.

13. Zhang L and Ai H: Effects of Gua Lou Gui Zhi decoction on c-fos and c-jun in epileptic rats. Sichuan Hua Xi Zhong Yi Yao Yan Jiu Suo 23: 21-22, 2005.

14. Hu H, Li Z, Zhu X, Lin R, Lin J, Peng J, Tao J and Chen L: Gua Lou Gui Zhi decoction suppresses LPS-induced activation of the TLR4/NF- $\kappa \mathrm{B}$ pathway in BV-2 murine microglial cells. Int $\mathrm{J}$ Mol 31, 1327-1332, 2013.

15. Hu H, Li Z, Zhu X, Lin R, Lin J, Peng J, Tao J and Chen L: GuaLou GuiZhi decoction inhibits LPS-induced microglial cell motility through the MAPK signaling pathway. In J Mol Med 32: 1281-1286, 2013.

16. Li ZF, Lin RH, Mao JJ, Hu HX, Zhu XQ, Chen WL and Chen LD: Protective effect of Gualou Guizhi decoction on BV-2 cells injured by glutamate. Journal of Fujian University of Traditional Chinese Medicine 23: 14-17, 2013.

17. Huang J, Tao J, Xue XH, et al: Gua Lou Gui Zhi decoction exerts neuroprotective effects on post-stroke spasticity via the modulation of glutamate levels and AMPA receptor expression. Int J Mol Med 31: 841-848, 2013.

18. Chen X, Li H, Huang M, Huang M, et al: Effect of Gua Lou Gui Zhi decoction on focal cerebral ischemia-reperfusion injury through regulating the expression of excitatory amino acids and their receptors. Mol Med Rep 10: 248-254, 2014.

19. Longa EZ, Weinstein PR, Carlson S and Cummins R: Reversible middle cerebral artery occlusion without craniectomy in rats. Stroke 20: 84-91, 1989.

20. Bederson JB, Pitts LH, Germano SM, Nishimura MC, Davis RL and Bartkowski HM: Evaluation of 2,3,5-triphenyltetrazoliumchloride as a stain for detection and quantification of experimental cerebralin farction in rats. Stroke 17: 1304-1308, 1986.

21. Hou SZ, Li Y, Zhu XL, Wang ZY, Wang X and Xu Y: Ameliorative effects of diammonium glycyrrhizinate on inflammation in focal cerebral ischemic-reperfusion injury. Brain Res 1447: 20-27, 2012.

22. Weiss N, Miller F, Cazaubon S and Couraud PO: The blood-brain barrier in brain homeostasis and neurological diseases. Biochim Biophys Acta 1788: 842-857, 2009.

23. Zhan $C$ and Yang J: Protective effects of isoliquiritigenin in transient middle cerebral artery occlusion-induced focal cerebral ischemia in rats. Pharmacol Res 53: 303-309, 2006.

24. Kape R, Parniske M, Brandt S, Werner D: Isoliquiritigenin, a strong nod gene- and glyceollin resistance-inducing flavonoid from soybean root exudate. Appl Environ Microbiol 58: 1705-1710, 1992.

25. Liu DZ, Xie KQ, Ji XQ, Ye Y, Jiang CL, et al: Neuroprotective effect of paeoniflorin on cerebral ischemic rat by activating adenosine A1 receptor in a manner different from its classical agonists. Br J Pharmacol 146: 604-611, 2005.

26. Mao QQ, Zhong XM, Feng CR, Pan AJ, Li ZY, et al: Protective effects of paeoniflorin against glutamate-induced neurotoxicity in PC12 cells via antioxidant mechanisms and $\mathrm{Ca}^{(2+)}$ antagonism. Cell Mol Neurobiol 30: 1059-1066, 2010.

27. Guo RB, Wang GF, Zhao AP, Gu J, Sun XL and Hu G: Paeoniflorin protects against ischemia-induced brain damages in rats via inhibiting MAPKs/NF- $\mathrm{BB}$-mediated inflammatory responses. PLoS ONE 7: e49701, 2012.

28. Kim SW, Lim CM, Lee HK and Lee JK: The use of Stronger NeoMinophagen C, a glycyrrhizin-containing preparation, in robust neuroprotection in the postischemic brain. Anat Cell Biol 44: 304-313, 2012.

29. Gong G, Xiang L, Yuan L, et al: Protective effect of glycyrrhizin, a direct HMGB1 inhibitor, on focal cerebral ischemia/reperfusion-induced inflammation, oxidative stress, and apoptosis in rats. PLoS One 9: e89450, 2014

30. Sun YX, Tang Y, Wu AL, Liu T, Dai XL, Zheng QS and Wang ZB: Neuroprotective effect of liquiritin against focal cerebral ischemia/reperfusion in mice via its antioxidant and antiapoptosis properties. J Asian Nat Prod Res 12: 1051-1060, 2012. 